\title{
Cellular Composition in the Aging Cerebral Cortex of Humans
}

\author{
V. P. Kalanjati, ${ }^{1}$ M. W. Hendrata, ${ }^{1}$ and F. N. Ardana ${ }^{1}$
}

Received July 2, 2019

\begin{abstract}
The numbers/densities of neurons and gliocytes and the neuron-to-glia ratios were calculated in different layers of the cerebral cortex of three subjects in a post mortem study. The subjects were 60 to 70 years old (which was qualified as a relatively early period of aging) and had no history of neurodegeneration diseases. The brains embalmed in $10 \%$ formalin-fixative solution were processed, and coronal $4-\mu \mathrm{m}-$ thick slices of tissues of the frontal, parietal, temporal, and occipital lobes were prepared and stained with hematoxylin-eosin. Clear cortical lamination was observed in all lobes. In the frontal lobe, the mean neuron density (number of neurons within visual fields at a $400 \times$ magnification) was significantly higher, while that of gliocytes was significantly lower compared to the respective values in other lobes $(P<0.05)$. A significant association between the number of neurons and glia was found in the frontal lobe $\left(\mathrm{r}^{2}=0.40, P=0.0029\right)$ and in all superficial cortical layers $\left(\mathrm{r}^{2}=0.72, P=0.0025\right)$, but not in the deeper layers $\left(\mathrm{r}^{2}=0.18, P=0.14\right)$. The mean neuron-to-glia ratio in different lobes and layers varied from $1.0: 0.81$ to $1.0: 3.54$. It is concluded that, in early-aging subjects with no cerebral pathology, the quantitative characteristics of cortical neurons and glial cells do not differ significantly from those in the brains of younger adult healthy subjects.
\end{abstract}

Keywords: neurons, gliocytes, neocortex, cortical zones, neuron-to-glio ratio, aging, humans

\section{INTRODUCTION}

In humans, the cerebral cortex consists of approximately $80 \%$ of the whole brain mass. According to early estimates, 10 to 16 billions of neurons have been observed in the cortex of adult human brains with a mean estimated mass of 1233 $\mathrm{g}[1]$, and the above numerical value for neurons is at present considered significantly underestimated. The human cerebral cortex can be divided phylogenetically into the allocortex and neocortex. The allocortex is formed first, which would then develop into the paleocortex and the archicortex. These include the olfactory cortex, dentate gyrus, and hippocampus per se (of the cornu Ammonis). The latter structure has a lesser number of laminae compared to the neocortex. Most regions of the latter contain six layers composed of different cell type patterns, namely the supragranular layer of laminae I-III, internal granular layer of lamina IV, and infragranular layer of laminae V-VI. In layers

\footnotetext{
${ }^{1}$ Faculty of Medicine, Universitas Airlangga, Surabaya, Indonesia. Correspondence should be addressed to V. P. Kalanjati (e-mail: viskasari-p-k@fk.unair.ac.id).
}

III and V, a part of the neurons possesses larger cell bodies, especially the pyramidal neurons of layer $\mathrm{V}$ at the motor area [1-3].

The cellular composition in distinctive cortical layers correlates with the network organization, and, thus, with the functions of specific areas. The numbers of neurons and gliocytes in the human brains have been a debatable subject for many years due to significant differences in both methodology and/or results obtained. The neuron-to-glia ratio has been reported to be smaller compared to what was observed previously [3-5]. In the aging brain, this would arguably change due to the degeneration process [1-3]. In 2050, the proportion of adults over 60 years old will be approximately $22 \%$ worldwide. The neurodegenerative disorders are expected to arise, in particular, with Alzheimer disease (AD) being one of the most common causes of dementia [4-6].

In most early studies, attention was focused more on the adult human brains (those of subjects younger than 60 years). The deeply aged brains and brains subjected to strong neurodegenerative modifications were also covered widely, while cellular compositions of the neocortex of healthy aging (but not aged) individuals have been described 
to a much lesser extent. This is why we analyzed in this study the neuron and glia numbers in different parts of the neocortex (frontal, parietal, temporal, and occipital lobes) of the aging human brains with no signs of cerebral pathology, to determine possible specific features of the cell composition and lamination in these regions; for this purpose, a standard histological technique was used.

\section{METHODS}

Brain tissue samples were obtain from three dead individuals aged 60 to 70 years. The cadavers were preserved using standard techniques in the Department of Anatomy and Histology, Faculty of Medicine, Universitas Airlangga (Surabaya, Indonesia). According to a detailed body of accessible medical information, all above subjects had no histories of any cerebral pathology, and their causes of death were not related to any neurological disease.

All bodies were subjected immediately after death to injection of a formalin (10\%) fixative solution via the femoral artery six months prior to the current study. As was shown, the respective samples of brain tissues are characterized by relatively good quality for a further histological study [7-9]. After removing the braincase, the whole encephalon was removed, processed for histology slides ( $4 \mu \mathrm{m}$-thick parasagittal slices), and stained with hematoxyllin-eosin using standard routine techniques. Three adjacent sections from each sample were analyzed in duplicate under a light microscope (Olympus, Japan) using a 400× magnification. Photomicrographs from each slide were taken with a digital camera (Nikon, Japan); numbers of neurons and gliocytes within test zones (visual fields at the above magnification) of the frontal, parietal, temporal, and occipital lobes of each slide were counted and analyzed using CellSens software (Olympus, Japan). The countings were done twice independently from 9 to 10 visual fields from each cortical region in each lobe.

The neurons were identified according to their shape and characteristics of their nuclei. The nucleus of a neuron was identified according to its oval, triangular, or round shape with pink or light-purple color; the pyknotic nuclei were darker. Oligodendroglia units were detected by their shape and small dark-purple nuclei; a layer of the cytoplasm could be distinguished with difficulties.
The nuclei of the microgliocytes and astrocytes were differentiated by their small dimensions, an openfaced, oval, or rounded form, or by the slightly larger nuclei with some processes, respectively [7-9].

The normality Shapiro-Wilk and Barttlet's homogeneity tests were done prior to the one-way ANOVA or Kruskal-Wallis test followed by either Tukey's or Dunn's post-hoc test, respectively (the latter, to seek significant differences between groups). The ratios of neurons and glial cells in four lobes were also compared and calculated. Simple linear regressions were calculated to test the association between neuron-to-glia numbers among four lobes and between the superficial and deeper cortical layers (MaxStat 3.06, Germany). The significance of intergroup differences was estimated at $P<0.05$.

\section{RESULTS}

Considering the experimental technique used in a post mortem study, it was difficult to expect very high quality of the obtained cortical slices. Nonetheless, it was found that it was possible to successfully differentiate neurocytes and gliocytes and to perform necessary calculations. We found that there were a moderate number of artefacts in nearly all slides observed; those were mostly composed of pyknotic nuclei of the neurons. The greatest average number (density) of neurons was observed in the occipital lobe, while the lowest respective index was met in the frontal lobe, and these data showed significant differences with respect to those of the temporal and occipital lobes $(P<0.001)$. The mean numbers of glia units were found to be least in the parietal lobe, while in the frontal lobe these cells were most abundant $(P<0.01)$. The regression coefficient of the neuron-to-glia ratio in the frontal lobe showed a significance with $P=0.0029$ and $r^{2}=0.40$ (Table 1).

In the superficial cortical layers I-III, the number of neurons in the frontal lobe was significantly lower when compared to that in other lobes $(P=0.01)$. There were significant differences in the neuron numbers in these superficial cortical layers between the frontal lobe to the temporal one $(P<0.05)$ and between the frontal lobe to the occipital one $(P<0.05)$. The number of gliocytes in the frontal lobe, however, was significantly greater when compared to those in other lobes $(P=0.013)$. 

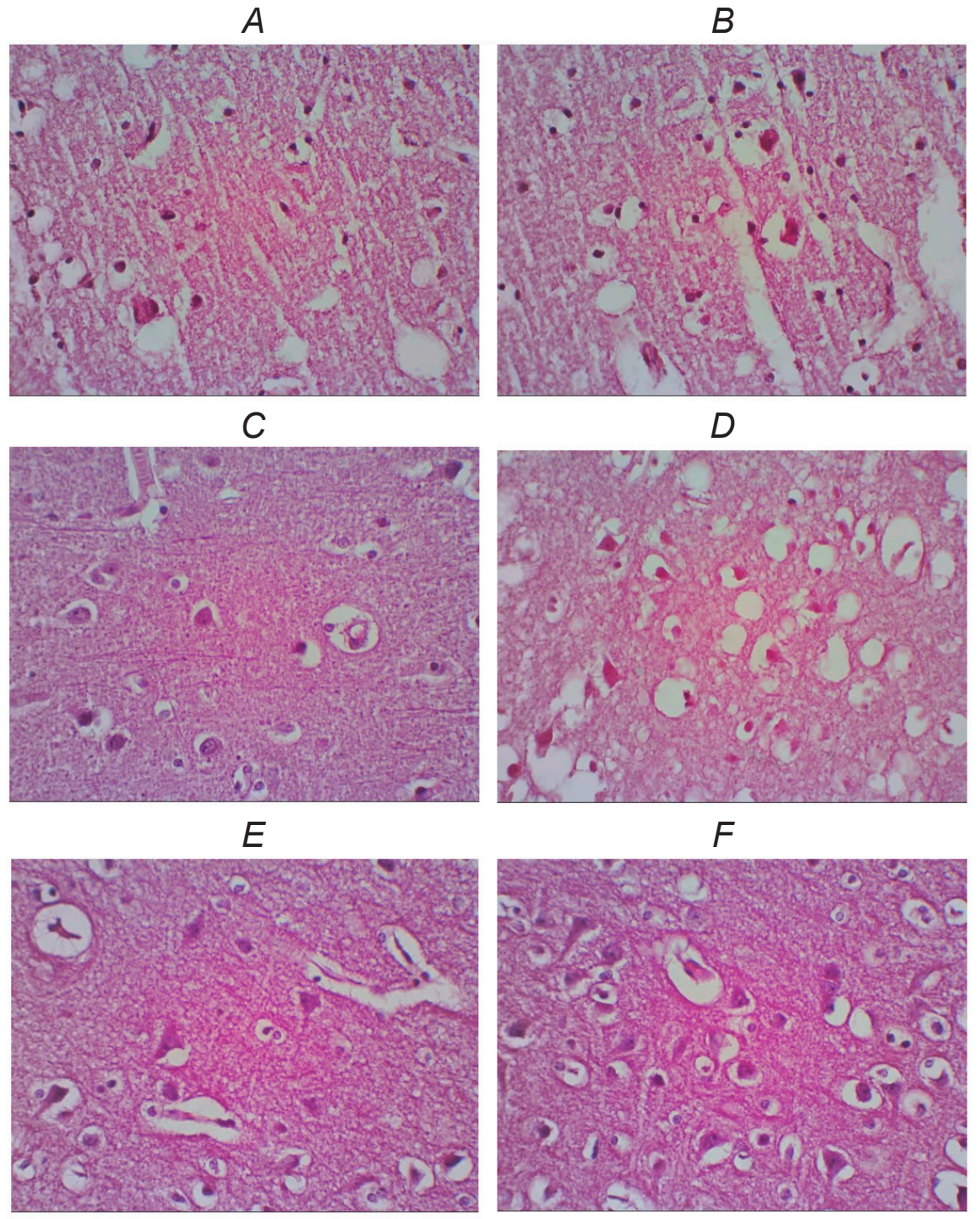

G

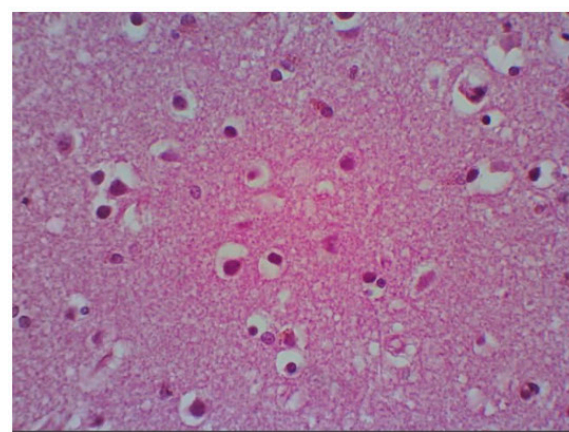

$H$

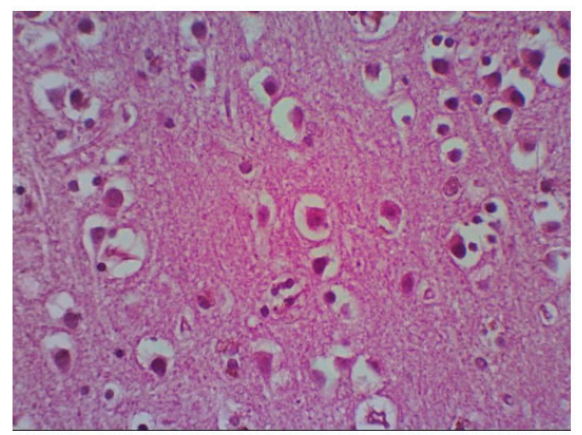

F i g. 1. Representative histological slices of superficial (left column) and deeper (right column) layers of the frontal (A, B), parietal (C, D), temporal (E, F), and occipital $(G, H)$ lobes of the neocortex. The scale bar in $G$ $(10 \mu \mathrm{m})$ is common to all photomicrographs. In $\mathrm{H}$, a neuronal cell with the large soma, pinkish-purple nucleus, and an apical dendrite is shown by arrow. Microglial cells are detected by a relatively small size of the nuclei, smaller size of the nuclei, while astrocytes have open-faced-type nuclei, in contrast with the dark purple nuclei of oligodendrocytes. 
T a b 1 e 1. Quantitative Analysis of Neurons and Glial Cells in Different Lobes of the Adult Early-Aging Human Brains

\begin{tabular}{|c|c|c|c|c|c|}
\hline \multicolumn{2}{|c|}{ Region } & Neurons & Gliocytes & Linear regression & Neuron-to-glia ratio \\
\hline \multirow{3}{*}{ Frontal lobe } & Layers I-III & $14.44 \pm 2.55$ & $24.67 \pm 3.10$ & $r^{2}=0.72, P=0.002^{5} \mathrm{e}$ & $1.0: 0.81$ \\
\hline & Layers IV-VI & $17.44 \pm 3.27$ & $21.56 \pm 3.57$ & $r^{2}=0.18, P=0.144$ & $1.0: 1.14$ \\
\hline & All layers & $15.94 \pm 2.04^{\mathrm{a}}$ & $23.11 \pm 2.32^{b}$ & $r^{2}=0.40, P=0.0029$ & $1.0: 0.98^{\mathrm{c}}$ \\
\hline \multirow{3}{*}{ Parietal lobe } & Layers I-III & $20.11 \pm 2.01$ & $12.22 \pm 0.91$ & & $1.0: 1.74$ \\
\hline & Layers IV-VI & $25.67 \pm 1.86$ & $13.11 \pm 0.87$ & & $1.0: 2.07$ \\
\hline & All layers & $22.89 \pm 1.49$ & $12.67 \pm 0.62$ & $r^{2}=-0.04, P=0.5881$ & $1.0: 1.91$ \\
\hline \multirow{3}{*}{ Temporal lobe } & Layers I-III & $24.67 \pm 2.15$ & $14.78 \pm 1.71$ & & $1.0: 2.00$ \\
\hline & Layers IV-VI & $34.11 \pm 4.15$ & $12.22 \pm 2.11$ & & $1.0: 3.54$ \\
\hline & All layers & $29.39 \pm 2.54$ & $13.50 \pm 1.36$ & $r^{2}=0.11, P=0.0955$ & $1.0: 2.77$ \\
\hline \multirow{3}{*}{ Occipital lobe } & Layers I-III & $25.78 \pm 3.03$ & $14.00 \pm 1.84$ & & $1.0: 2.12$ \\
\hline & Layers IV-VI & $33.22 \pm 4.38$ & $18.56 \pm 1.53$ & & $1.0: 1.94$ \\
\hline & All layers & $29.50 \pm 2.74$ & $16.28 \pm 1.29$ & $r^{2}=0.02, P=0.2756$ & $1.0: 2.03$ \\
\hline
\end{tabular}

Footnotes: Mean total numbers \pm s.e.m. within test-zones (fields of vision at $\times 400$ ) are shown; ${ }^{a}$ one-way ANOVA, $\mathrm{P}=0.0001$; ${ }^{\mathrm{b}} \mathrm{Kruskal}-$ Wallis, $\mathrm{P}=0.001 ;{ }^{\mathrm{c}}$ Kruskal-Wallis, $\mathrm{P}=0.0009$.

In the deeper cortical layers IV-VI, the frontal lobe was characterized by a significantly lower number of neurons compared to those in other lobes $(P=0.0073)$, while the glia number was significantly greater when compared to those of other lobes $(P<0.005$; Table 1). The regression coefficient of the neuron-to-glia ratio in the superficial layers $\left(\mathrm{r}^{2}\right)$ was equal to $0.72(P=0.0025)$; in the deeper layers $r^{2}$ was only $0.18(P=0.14)$.

The mean neuron-to-glia ratio in all lobes corresponded to $1.0: 1.92$. The least ratio was observed in the frontal lobe (in layers I-III, $1.0: 0.81$; in all layers of this lobe, $1.0: 0.98$ ), while the largest was found in the temporal lobe (layers IVVI, $1.0: 3.54$; in all layers, $1.0: 2.77$ ), (Table 1 ).

\section{DISCUSSION}

In this study, we found that, in all lobes of the human neocortex, neurons are more numerous in deeper cortical layers (IV-VI) when compared to the superficial layers I-III. The number (density) of neurons was greater in the occipital and temporal lobes than in the frontal and parietal ones; the number of gliocytes was significantly greater in the frontal lobe. A significant association between lower neuron numbers and higher numbers of glial cells in the frontal lobe was arguably correlated, especially in the superficial layers. In the current study, the mean neuron-to-glia ratio varied approximately from 1.0 : lesser than 1.0 to 1.0 : more than 3.5 . The smallest ratios were found in the frontal lobe, while the highest ratios were observed in the temporal lobe. In the previous study on adult human cortices (those of younger subjects), this ratio was found to be close to $1.0: 10.0$, i.e., much larger than what was found in our observations. However, in other more recent studies using comparable techniques, this ratio between neurons and the gliocytes was reported to be close to $1.0: 1.0[2,3,11]$.

In humans, the cellular cortical lamination is arranged in both horizontal and vertical (or radial, columnar) networks [9-11]. Using magnetic resonance microscopy at $9.4 \mathrm{~T}$, a highly specific horizontal pattern of lamination in the human cortical brain, including the neocortex, was shown [9-13]. A specific pattern of cortical lamination has been reported to correspond to unique predominant functions in each area. These arrangements are by no means exclusive; on the other hand, they are interconnected with each other and, of course, connected to other regions of the CNS [10-12]. The signaling molecules, such as FGFs (fibroblast growth factors), Wnts (wingless ones), and BMP (bone morphogenetic protein), were reported to be mostly responsible for regulation of the position and size of the cortical region [11-13]. The columnar cytoarchitecture of neurons in the neocortex may be related to the discrete connectivity to other cells and correlated with a specific functional work.

The study of cortical areas related to the whiskers (vibrissae) in rodents and carnivores showed that an individual whisker has a specific cluster of synapses between specific thalamic axons and neurons in layer IV of the somatosensory cortex, which 
received sensory information from the whisker follicles via the brainstem to the thalamus. From layer IV, the signals then mostly spread vertically within a column into layers II-III to be processed and then integrated with the horizontal transmission to produce the output via layer-V neurons. This delineates a specific organization and topographic map in certain cortical area, which corresponds to a specific area in the rodents' face $[4,10,12]$. Obvious parallels to such organization were found in the primary visual cortex. These unique cytoarchitectural cellular compositions exhibit some variations depending on their functions. Such "vertical" structural networks are characterized by relatively uniform neuron-to-glia ratios.

Neurons are believed to develop within the piaoriented layers of the cerebral cortices within the end of the first month of pregnancy; these would then be followed by the generation of neurons in the transient proliferative embryonic zones, i.e., ventricular and subventricular zones (VZ and SVZ, respectively); the latter are located close to the former. These neurons will migrate into the neocortex area via radial and tangential paths across the intermediate zone. Each newer generation of these postmitotic neurons will bypass the older and deeper located neurons, following an inside-out gradient of neurogenesis [11-13].

Two types of functionally different cortical neurons are, in principle, distinguished, namely projection neurons and interneurons. The latter are further classified into spiny pyramidal and aspiny nonpyramidal cells [10-13]. The spiny units are believed to have mostly glutamatergic modality. They, in many cases, reside in layer IV, whereas the aspiny units are GABAergic neurons distributed widely in all layers. Examples of projection cortical neurons are cortico-pontine and cortico-tectal cells that are considered associative projecting neurons. Other projecting neurons are commissural projecting ones that send their axons to synapse with the correlated region of the opposite hemisphere. All these arrangements are characterized by relatively specific neuron-to-glia ratios [10-13].

Significant alterations of the above-mentioned patterns were reported to be manifested under several conditions, first of all at certain neurodegenerative ones, including Alzheimer's disease (AD). The process of physiological aging has been also supposed to be related to certain shifts in the arrangement of cell ensembles in different cortical zones. There is, however, limited information on the extent of such shifts within a relatively early presenile period at the normal (physiological) course of the aging process, when persons of the respective age preserve a sufficiently high level of behavioral and cognitive activities comparable to that in younger adult individuals.

However, rather than neuronal and/or glial loss, the cognitive and memory declination in $\mathrm{AD}$ is more correlated with molecular and subtle structural impairments. For example, in the hippocampal perforant pathway, N-methyl-D-aspartate (NMDA) receptor expression levels are altered, and, thus, the circuit does not function well. The imbalance between the excitatory and inhibitory synapses would disrupt the functionality of the physiological network [13-15]. Impaired dendritic arborization in the hippocampal circuits of aging humans and rodents has also been reported, and this might be correlated with the attention deficits observed among elderly patients of AD [14-16].

The role of estrogen as a "preservation" agent against degeneration has been suggested, as the estrogen receptors (ERs) are widely distributed in the brain. Epidemiology studies reported that the sex steroid hormone levels demonstrate certain correlations with the prevalence of Alzheimer's and Parkinson's diseases. It was reported that inhibition of the aromatase enzymes providing lower estrogen levels is correlated with altered amyloid deposition and more severe strokes in AD. Estrogen is reported to act at the mitochondrial level as it regulates the transcription of mitochondrial transcription factor A (TFAM), starting the transcription and replication of mtDNA (mitochondrial DNA) [14-16]. Testosterone and progesterone have also been reported to serve as neuroprotective agents via several pathways including lower susceptibility to oxidative stress and ability to protect the dendritic spines related to the BDNF (brain-derived neurotrophic factor) expression and to regulate the growth of Purkinje cells and oligodendrocytes via the activation of the MAPK/ERK and P13K/Akt pathways [14-16] and, thus, to modulate the neuron-to-glia ratio. It was reported that clumps of pyramidal cells in the neocortex are more prone to neurofibrillary tangle formation and the degenerative process than those of the inhibitory interneurons. The association fibers interconnecting regions on a hemisphere are at a higher risk of this degeneration, as well as the cholinergic projection from the nucleus basalis of Meynert, although the thalamocortical projection 
fibers would less susceptible $[6,7,14]$.

It is obvious that, from the beginning of aging, all the above-mentioned processes start to affect the interrelations between neuronal and glial cells in the brain and even the spatial interrelations between cortical layers. The question is the following: What is the extent of the respective modifications within the early stages of physiological (normal) aging in the absence of neurodegenerative pathologies?

Considering the results of our study, we come to the following conclusion. The composition of neurons and gliocytes (in particular, the neuronto-glia ratio) in the brain of subjects within the relatively early stages of normal aging (with no noticeable signs of neurodegenerative diseases) demonstrates no principal deviations from the pattern observed in younger adult human cortices with no major pathology observed. Of course, further functional studies should be combined with morphological ones to detect possible changes in the structure and activity of cortical neuronal networks and cortico-glial ensembles related to the early stages of the aging process.

Obvious limitations of our study should be mentioned. First, this is related to the nature of the study per se (post mortem one). Second, the volume of the studied group is highly limited (three subjects). Third, we have not examined a control group of comparable age and one should compare our results exclusively with the data published earlier.

Acknowledgement. Our thanks to the entire staff and Head of the Department of Anatomy and Histology, Faculty of Medicine, Universitas Airlangga, Surabaya, Indonesia.

This study was supported by the Faculty of Medicine, Universitas Airlangga, Surabaya, Indonesia.

This is a post mortem study. Written permissions were obtained from the relatives of the persons whose brains were subjected to examination. All procedures were in accordance with the ethical standards of the institutional and/or national Research Committees, in particular, of the Ethic Committee of the Faculty of Medicine, Universitas Airlangga (No. 42/ KEPK/ FKUNAIR) and with the 1964 Helsinki declaration and its later amendments or comparable.

The authors of this work, V. P. Kalanjati, M. W. Hendrata, and F. N. Ardana, confirm the absence of any conflicts regarding commercial or financial relations, relations with organizations or persons that could in any way be connected with the research, as well as co-author relationships.

\section{REFERENCES}

1. A. Araque and M. Navarrete, "Glial cells in neuronal network function," Philos. Trans. R. Soc. Lond. B, Biol. Sci., 365, No. 1551, 2375-2381 (2010).

2. C. S. von Bartheld, J. Bahney, and S. Herculano-Houzel, "The search for true numbers of neurons and glial cells in the human brain: a review of 150 years of cell counting," J. Comp. Neurol., 524, No. 18, 3865-3895 (2016).

3. S. Herculano-Houzel, "The glia/ neuron ratio: how it varies uniformly across brain structures and species and what that means for brain physiology and evolution," Glia, 62, No. 9, 1377-1391 (2014).

4. C. C. Sherwood, C. D. Stimpson, M. A. Raghanti, et al., "Evolution of increased glia-neuron ratios in the human frontal cortex," Proc. Natl. Acad. Sci. USA, 103, No. 37, 13606-13611 (2006).

5. R. C. Pearson, M. M. Esiri, R. W. Hiorns, et al., "Anatomical correlates of the distribution of the pathological changes in the neocortex in Alzheimer disease (neurofibrillary tangles/ lamination/ clustering)," Proc. Natl. Acad. Sci. USA, 82, No. 13, 4531-4534 (1985).

6. R. A. Cromarty, J. Schumacher, S. Graziadio, et al., "Structural brain correlates of attention dysfunction in Lewy body dementias and Alzheimer's disease," Front. Aging Neurosci., 10, 347 (2018).

7. S. Chatterjee, "Artefacts in histopathology," J. Oral Maxillofac. Pathol., 18, Suppl. 1, S111-S116 (2014).

8. R. H. Garman, "Histology of the central nervous system," Toxicol. Pathol., 39, No. 1, 22-35 (2011).

9. V. P. Kalanjati, A. K. Dewi, and M. W. A. Santosa, "Quantitative study on human cerebellar cortex from anatomy cadaver preparations," Int. J. Morphol., 35, No. 1, 167-171 (2017).

10. J. H. Lui, D. V. Hansen, and A. R. Kriegstein, "Development and evolution of the human neocortex," Cell, 146, No. 1, 18-36 (2011).

11. B. Mota and S. Herculano-Houzel, "All brains are made of this; a fundamental building block of brain matter with matching neuronal and glial masses," Front. Neuroanat., 8, 127 (2014).

12. G. M. Fatterpekar, T. P. Naidich, B. N. Delman, et al., "Cytoarchitecture of the human cerebral cortex: MR microscopy of excised specimens at 9.4 Tesla," AJNR Am. J. Neuroradiol., 23, No. 8, 1313-1321 (2002).

13. Z. Molnár, G. J. Clowry, N. Šestan, et al., "New insights into the development of the human cerebral cortex," $J$ Anat., 235, No. 3, 432-451 (2019).

14. D. E. Bergles, R. Jabs, and C. Steinhäuser, "Neuron-glia synapses in the brain," Brain Res. Rev., 63, Nos. 1-2, 130-137 (2010).

15. J. H. Morrison and P. R. Hof, "Life and death of neurons in the aging brains," Science, 278, No. 5334, 412-419 (1997).

16. S. Zárate, T. Stevnsner, and R. Gredilla, "Role of estrogen and other sex hormones in brain aging, neuroprotection and DNA repair," Front. Aging Neurosci., 9, 430 (2017). 